\title{
Bilateral sequential lung transplantation: technical aspects
}

\author{
Haytham Elgharably, Michael J. Javorski, Kenneth R. McCurry \\ Department of Thoracic and Cardiovascular Surgery, Cleveland Clinic Foundation, Cleveland, OH, USA \\ Contributions: (I) Conception and design: All authors; (II) Administrative support: None; (III) Provision of study materials or patients: None; (IV) \\ Collection and assembly of data: None; (V) Data analysis and interpretation: None; (VI) Manuscript writing: All authors; (VII) Final approval of \\ manuscript: All authors. \\ Correspondence to: Kenneth R. McCurry, MD. Department of Thoracic and Cardiovascular Surgery, Cleveland Clinic, 9500 Euclid Avenue/Desk J4-1, \\ Cleveland, OH 44195, USA. Email: MCCURRK@ccf.org.
}

\begin{abstract}
The surgical technique for lung transplantation has evolved dramatically over the last three decades. Significant improvements in short term outcomes in the early years of lung transplantation were due, in large part, to techniques developed to reduce airway anastomotic complications in single lung transplantation. Following development of the technique of en bloc double lung transplantation, evolution to the bilateral sequential technique further reduced airway complications for double lung transplantation. More recently, some programs have utilized the en bloc double lung transplant technique with bronchial artery revascularization to aid airway healing and potentially improve short- and long-term outcomes. The experience with bronchial artery revascularization remains limited to a few series, with the technique having not been widely adopted by most lung transplant programs. With the implementation of priority allocations schemes in many countries, patients with higher risk profiles are being prioritized for transplantation which results in more complex procedures in fragile recipients with multiple comorbidities. This includes the increased need for concomitant cardiac procedures as well as performing lung transplantation after prior cardiothoracic surgery. Different surgical approaches have been described for bilateral sequential lung transplantation with or without intra-operative mechanical circulatory support (MCS), such as sternotomy, clamshell (bilateral anterior thoracotomies with transverse sternotomy), and bilateral thoracotomy incisions. Herein, we aim, not only to describe the various surgical approaches for double lung transplantation, but to provide a comprehensive review of other aspects related to the recipient pathology and different anatomical variants as well as handling technical challenges that might be encountered during the procedure.
\end{abstract}

Keywords: Lung transplantation; bilateral sequential; surgical technique

Submitted Dec 28, 2020. Accepted for publication Jun 02, 2021.

doi: $10.21037 /$ jtd-2021-22

View this article at: http://dx.doi.org/10.21037/jtd-2021-22

\section{Introduction}

The first successful bilateral lung transplantation (LTx) was performed in 1988 (1), five years after the first successful single LTx (2). Initially, bilateral LTx was performed en bloc through median sternotomy; however, this procedure was complex and had a high rate of tracheal anastomotic ischemic complications. This prompted modification of the technique into bilateral sequential (BS)-LTx as first proposed by Dr. Pasque in 1990, which soon after became the preferred method for bilateral LTx due to the lower risk of airway ischemia (3). Moreover, experience with BS-LTx continued to grow and has become the current dominant mode of LTx over single LTx (4). The most recent data from the International Society for Heart and Lung Transplantation (ISHLT) reports that in 2017, there were 3,626 adult bilateral and 826 single LTx (4). The preference for bilateral versus single lung transplant is, in part, based on an observed increase in long term survival advantage (5). With the increase in case volume, different surgical approaches of BS-LTx have evolved over the years (6). This article will discuss the different approaches, 
surgical technique, disease-specific considerations, and unique anatomic variants in the context of BS-LTx.

\section{Surgical approaches for bilateral sequential lung transplantation}

The patient is placed supine with arms tucked at the patient's side. A roll is placed longitudinally underneath the spine for clamshell or bilateral thoracotomy incisions, while transversely underneath the shoulders for sternotomy. The neck is prepped into the field along with the chest, abdomen and bilateral groins to provide vascular access for intraoperative or postoperative mechanical circulatory support (MCS) if needed. Intra-operative MCS that can be used includes cardiopulmonary bypass (CPB) and extracorporeal membrane oxygenation (ECMO); venoarterial (VA) or venovenous (VV). Surgical approaches for BS-LTx varies from sternotomy, bilateral thoracotomy, to clamshell (bilateral thoracosternotomy) incisions based on the surgeon preference, intra-thoracic pathology, and anatomical considerations. For instance, we prefer clamshell approach for cystic fibrosis, suppurative lung disease, and redo LTx or history of thoracic procedure. For favorable anatomy, obstructive or fibrotic lung disease cases, our approach is bilateral thoracotomy incisions. For bilateral LTx with bronchial artery revascularization, sternotomy is our preferred approach. Sternotomy or clamshell incision can be utilized for BS-LTx with concomitant cardiac procedure and pulmonary hypertension.

\section{Clamshell incision}

The approach for clamshell incision is typically though the $4^{\text {th }}$ intercostal space. For females an inframammary incision is made, with dissection of the subcutaneous tissue to reach the fourth intercostal space. Upon entering the intercostal space, the internal mammary artery and vein are identified and ligated on each side. The sternum is separated at the level of the $4^{\text {th }}$ intercostal space. Boasquevisque et al. (7) described creating the transverse sternotomy at an oblique angle going cranial to caudal to help prevent sternal overriding. Retractors may then be placed. This incision offers the best exposure and allows rapid access for central cannulation if there is unexpected hemodynamic compromise. With the clamshell approach, both pleura are opened and the pericardium may be opened anteriorly to aid in dissection of the hilum and to allow manipulation of the heart.

\section{Bilateral thoracotomy}

Another option for surgical approach is a sternal sparing bilateral anterior thoracotomy. This approach has been shown to avoid sternal complications associated with the clamshell approach, along with providing increased respiratory function in the early postoperative period, decreased operative time, and decreased intensive care unit stay (8). The approach is an anterolateral incision at the $4^{\text {th }}$ intercostal space. While the skin incision can be smaller, the intercostal space is opened from about the level of the internal mammary artery anteriorly to the posterior end of the rib to allow rib spreading as needed. Many surgeons prefer to minimize rib spreading to diminish postoperative pain. The serratus anterior muscle can be divided or spared (muscle sparing thoracotomy), however the latissimus dorsi is generally spared. Entry into the $5^{\text {th }}$ intercostal space instead of the $4^{\text {th }}$ on the left side may sometimes provide better exposure of the left atrium, depending on pulmonary pathology (9).

\section{Sternotomy}

Median sternotomy is another approach for BS-LTx. This approach has been shown to have a lower risk of chest wall complications compared to the clamshell approach, likely due to preservation of the internal mammary arteries and improved early chest wall mechanics $(10,11)$. This approach can be considered in patients with minimal pleural adhesions and appropriate hilar depth. The feasibility can be predicted from preoperative CT imaging measuring the hilar depth and degree of pleural thickening (10). Elde et al. (10) reserved the median sternotomy approach for patients with left main bronchus depth less than $12 \mathrm{~cm}$ and a midline to cardiac apex distance of less than $10 \mathrm{~cm}$. The major downside for using a median sternotomy is that intra-operative MCS (CPB or VA ECMO) is typically needed for hemodynamic support while manipulating the heart for exposure of the hilar dissection and anastomosis. Thus, patients with a high likelihood of requiring intraoperative MCS during LTx, such as patients with primary pulmonary hypertension or significant secondary pulmonary hypertension, should be considered for sternotomy (12). In general, we have a strong preference for sternotomy in patients who have small pleural spaces and also have significant pulmonary hypertension. Other disease types that have been described as good candidates for median sternotomy approach are patients with COPD (depending 
on hilar depth) or pulmonary fibrosis, since there are usually minimal pulmonary adhesions; however, this may come with increased risk of bleeding and early graft dysfunction compared to thoracotomy incision without usage of MCS.

\section{Surgical technique of sequential bilateral lung transplantation}

For any surgical approach, pleural adhesions are taken down with electrocautery with extra care to not injure the native lung. A significant air leak may make ventilation difficult and may force use of unplanned intraoperative MCS. The native lung and hilum should be dissected before the donor lung arrives. The decision of which side to transplant first can be dependent upon preoperative ventilation/perfusion scan, if a significant difference in perfusion is present. Surgeon preference obviously may also influence the decision, as well. If a significant difference in perfusion exists, the side with the worse function is usually the lung which is transplanted first. If the LTx procedure is performed without CPB (i.e., either without any form of MCS or with VA or VV ECMO), single lung ventilation (with a double lumen endotracheal tube or, less commonly, a bronchial blocker) is used. However, if the patient is on full support with $\mathrm{CPB}$, some centers choose to explant both lungs and then implant the donor lungs sequentially to then be able to reperfuse both lungs at the same time (13). Obviously, if VV ECMO is utilized for intraoperative support, the ability to isolate lung ventilation is necessary. With intraoperative VA ECMO, we use a double lumen endotracheal tube and continuously ventilate at least one lung with low tidal volume.

Dissection starts by dividing the inferior pulmonary ligament at the diaphragm to the level of the inferior pulmonary vein. Hilar dissection is carried out to expose the pulmonary veins, PA, and bronchus. Care must be taken not to injure the phrenic nerve. Before proceeding with the pneumonectomy, particularly if pulmonary hypertension is present and CPB or VA ECMO not used, a PA occlusion test should be done in order to ensure hemodynamic stability; otherwise MCS may be indicated if PA pressures rise causing right ventricular dysfunction and hemodynamic instability. With the donor lung in the room, the pneumonectomy is performed in the standard fashion. The PA and pulmonary veins are encircled and a vascular stapler (or ligature, if preferred) is used to divide each one sequentially. It is important to remember to check for the PA catheter before firing the stapler across the vessel. The hilar vessels are divided as peripheral as possible to ensure enough length. Silk stay sutures may be placed through the staple line to give leverage to allow manipulation of the stapled hilar vessels and better visualization. The bronchus is divided just proximal to the upper lobe bronchus; first the cartilaginous wall of the bronchus is cut with a scalpel. Then the superficial layer of the membranous portion of the bronchus is incised with a scalpel, bronchial vessels are controlled and divided, then the outer layer is separated with electro-cautery.

After the lung is removed from the chest, the pericardium is cleared around the hilar structures to provide adequate mobilization for anastomosis. On the left, better exposure can be created by clearing the attachments from the left atrium to the PA and pericardium. On the right, the interatrial groove can be developed to better expose the left atrium, however, the anterior wall of the left atrium can become quite thin with this maneuver and caution is advised. The pulmonary arteries must be dissected proximally within the pericardium on the right, however, adequate length can many times be achieved on the left without entering the pericardium. Particular attention must be paid to avoid injury of the phrenic nerve on the right side and the recurrent laryngeal nerve on the left side, especially in case of significant adhesions in the mediastinum. It is important to obtain hemostasis of the surrounding structures, especially the posterior mediastinum because these will be difficult to visualize after implantation. Care should be taken to preserve as much peribronchial tissues as possible to reduce risk of ischemic complications of the airway anastomosis.

The order of implantation is main bronchus, PA and then left atrial cuff anastomosis. Prior to the first anastomosis, the bronchus is cleared of any secretions which are sampled for culture. It is our routine to irrigate the pleural cavity with antibiotic solution when the native lungs are colonized with multidrug resistant organisms. The donor lung is then placed into the thoracic cavity on iced laparotomy pads (some surgeons use a cooling pad). The bronchial anastomosis is performed with a running technique with 4-0 suture, starting at one corner and running along the posterior membranous portion to the opposite edge. The other end of the suture will then be used to run the anterior, cartilaginous portion. Attention is paid to not manipulate, as feasible, the donor bronchus with forceps and to avoid stripping tissue off the donor bronchus-both to preserve blood supply. Many surgeons use absorbable, braided suture while some utilize monofilament (polypropylene). Mild 
size mismatch can be overcome by adjusting the spacing of the suture line. For significant size mismatch, a telescoping technique can be used. After the airway anastomosis is complete, it is evaluated with video bronchoscopy by the anesthesia team.

The recipient $\mathrm{PA}$ is then clamped proximally and the staple line is excised. The donor PA is trimmed to an adequate length to prevent kinking. A 5-0 Prolene is started in the superior aspect and continued in a running fashion. We prefer to fold the posterior wall in the suture line (creating double thickness) to aid in hemostasis. The $\mathrm{PA}$ is left clamped until flushing can be completed after the last anastomosis. In the case of sternotomy with $\mathrm{CPB}$ (when required), we place a vent in the main PA which abrogates the need to clamp the left and right PAs allowing an open technique. Lastly, the left atrial cuff anastomosis is performed. While the pulmonary veins are retracted, a Satinsky clamp is placed on the body of the left atrium to allow for an adequately sized atrial cuff. The staple lines on the pulmonary veins are removed in a manner that creates a common orifice of atrial tissue. A 4-0 Prolene is sewn in a running fashion to create an endothelial to endothelial anastomosis of the donor and recipient atrial cuffs (by everting the suture line). Before the anastomosis is completed anteriorly, the last few sutures are left loose for de-airing prior to closure. The atrial cuff anastomosis is flushed with saline first, then the PA clamp is partially released to allow gradual reperfusion of the graft. The left atrial cuff clamp is released to flush air out. The atrial cuff anastomosis is tied down. Perfusion of the lung is carefully controlled, by progressively releasing the PA clamp over a period of $\sim 10$ minutes (in the absence of $\mathrm{CPB})$. Reperfusion of the new lung graft is a critical point of the procedure that requires preparation from the operative team for hemodynamic changes. There should be clear communication between the surgical and anesthesia teams. After checking hemostasis of the vascular suture line and allowing time for the graft to warm, ventilation is started with lung protective volume and pressures, on low $\mathrm{FiO}_{2}$. After ensuring satisfactory function of the lung graft and stable hemodynamics, the contralateral side pneumonectomy can be started. If performing bilateral anterolateral thoracotomies, the first thoracotomy is left open until both lung anastomoses are complete. The leftand right-side use the same implantation technique. The left side may sometimes be more challenging since it can be harder to expose the left atrial cuff anastomosis.

\section{Technical considerations related to recipient pathology}

\section{Fibrotic lung disease}

Since the introduction of lung allocation score (LAS) in the United States, the percentage of patients with fibrotic lung disease receiving LTx has increased significantly and

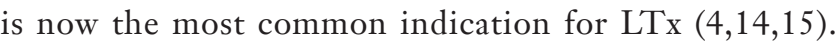
Thirty years ago, the great majority of pulmonary fibrosis patients received single LTx; now the majority of these patients are receiving bilateral LTx due to better survival trends $(4,15)$. There are some operative differences to consider when performing a BS-LTx in patients with fibrotic lung disease. Many of these patients will have a shrunken thoracic cavity and narrow operative field, for which a clamshell approach can be considered if there is concern for adequate exposure (12). Also, to aid in exposure for patients with small thoracic cavities, a figure of eight stitch can be placed in the dome of the diaphragm (central tendon), brought out of the chest at the most inferior and antero-lateral aspect of the cavity, and secured externally. This "diaphragm retraction stitch" can significantly aid exposure in small pleural cavities. Patients with fibrotic lung disease typically are more likely to have comorbidities like pulmonary hypertension with or without right ventricular dysfunction and coronary artery disease; therefore, there may be an increased need for intra-operative MCS, as these patients may not tolerate single lung ventilation $(7,16)$.

\section{Obstructive lung disease}

Chronic obstructive pulmonary disease was the leading diagnosis for patients receiving LTx for several decades, however, since LAS implementation it has become second to fibrotic lung disease (4). These patients may receive bilateral or single LTx; however, initially in the 1970s, only bilateral LTx was performed due to the issues encountered with the drastic difference of compliance in the native emphysematous lung and the transplanted lung (17). Advancements in LTx care has allowed us to mitigate these issues; however, BS-LTx is still the preferred approach today for obstructive lung disease. In cases of bullous emphysema, BS-LTx is the optimal approach, especially with a diagnosis of alpha-1 antitrypsin deficiency (18). Technically, incision at the $5^{\text {th }}$ intercostal space, as opposed to the $4^{\text {th }}$ for fibrotic lung disease, is recommended by some centers when using clamshell approach (19). These patients may also be good 
candidates for bilateral thoracotomy incision or median sternotomy since they typically have minimal adhesions.

\section{Suppurative lung disease}

Patients with suppurative lung disease like bronchiectasis and cystic fibrosis typically receive bilateral LTx since both lungs are typically colonized, and implanting a single allograft will have a great risk of becoming infected by the native contralateral lung (13). Due to expected pleural adhesions in this patient population, as a result of recurrent infections or prior thoracic procedures, we commonly use a clamshell incision although many times begin with bilateral thoracotomies to determine feasibility of accomplishing the operation without sternal transection. The $4^{\text {th }}$ intercostal space may be used; however, some centers recommend using the $5^{\text {th }}$ intercostal space on patients with suppurative lung disease $(8,19)$. Venuta et al. reports that a bilateral anterior thoracotomy approach is still possible in this patient population, even if they have had major thoracic procedures and/or expected pleural adhesions (8). Due to the difficultly of reaching any posterior and apical pleural adhesions present, an approach through median sternotomy is not recommended. Careful hemostasis must be obtained at the hilum for these patients, since there may be enlarged lymph nodes and bronchial arteries (7). While there are limited data, in the case of difficult to treat bacteria (e.g., Burkholderia species), we prefer to perform bilateral pneumonectomies on MCS and irrigate the residual tracheobronchial tree and pleural spaces prior to beginning lung graft implantation. In the case or other organisms, we still think it is important to irrigate the pleural cavities with antibiotic solution prior to implantation.

\section{Pulmonary hypertension}

Patients with a primary diagnosis of idiopathic pulmonary arterial hypertension represent a small percentage of all patients receiving LTx (4). Survival after bilateral LTx has been shown to be superior to single LTx for patients with primary pulmonary hypertension (20). In light of better reported outcomes of bilateral LTx for pulmonary hypertension compared to single LTx, BS-LTx is the preferred approach for these patients (21). The functional status of the right ventricle is a key aspect of evaluation of these patients. A dysfunctional right heart typically recovers after bilateral LTx; however, if the dysfunction is severe (e.g., requiring preoperative inotropic support) and irreversible the patient should be considered for heart-lung transplant. Allocation issues may play a role in the decision of double lung versus heart-lung transplantation. From a technical standpoint, intra-operative MCS should be utilized during the operation since the patient is not likely to handle single lung ventilation $(7,13)$. The surgical approach should then reflect the need to obtain access and cannulation for MCS; commonly clamshell or sternotomy is used.

\section{Prior cardiothoracic surgery}

Patients presenting for LTx may have undergone prior cardiac or thoracic procedures, especially in the era of LAS which has prioritized older age recipients with pulmonary fibrosis and potentially multiple comorbidities (22). Prior cardiothoracic procedures could include pleurodesis, nonanatomical or anatomical lung resection, coronary artery bypass grafting (CABG), or valve surgery. Shigemura et al. have shown in their analysis that pleurodesis and prolonged $\mathrm{CPB}$ were associated with an increased risk of mortality (23). McKellar et al. reviewed the United Network for Organ Sharing (UNOS) database for LTx after CABG and they reported increased mortality for bilateral LTx after CABG, but not for single LTx (24). In our experience, there was no significant difference in outcomes between LTx after prior cardiothoracic procedure when compared to a group of patients that underwent LTx without prior cardiothoracic procedure (25). Mild pleural adhesions can be dissected out with electrocautery or sharp dissection. For extensive pleural adhesions, e.g., after pleurodesis, that involve the majority of the lung surface, dissection can be carried out through an extra-pleural plane. Our preferred approach is to achieve hemostasis using the bipolar sealer Aquamantys ${ }^{\mathrm{TM}}$ (Medtronic, Minneapolis, MN, USA). For LTx after prior cardiac surgery, our preference would be bilateral thoracotomies to avoid prolonged operative time by adding a mediastinal dissection phase. Intra-operative MCS can be achieved through the groin vessels or axillary artery. In cases of prior CABG with patent grafts, extra caution is needed during pleural dissection near the mediastinum. Thus, adequate preoperative imaging is necessary for proper planning. Patients do not get evaluated for LTx at our program if they require a redo CABG (and are not candidates for percutaneous coronary intervention), given the complexity imposed on the procedure and concerns for long-term outcomes. Trans-catheter valve therapy provides a less invasive alternative for patients in need of redo valve surgery with LTx. 


\section{Tackling technical challenges}

\section{Pulmonary artery pathology}

Fragile PA is an expected scenario during LTx for pulmonary hypertension as well as older age recipients with chronic steroid use. Gentle manipulation of the PA is critical to avoid dissection of the vessel or disastrous injury during mobilization of the vessel, traction with instruments, or clamping. An additional challenge in the setting of fragile PA is size mismatch between donor and recipient PA. Sakamaki et al. reported a case of PA dissection early postLTx for pulmonary hypertension that required re-operation to redo the anastomosis (26). We prefer to imbricate the posterior wall suture line to aid in hemostasis with gentle pull on the sutures. It is always wise to be prepared for MCS cannulation in cases of fragile PA while the procedure is started off pump. In case of significant injury to the PA, options for reconstruction are autologous tissue, donor tissue (if available), prosthetic graft, and homograft reconstruction. A patch of pericardium (autologous or bovine) can be tailored into a tube and used for reconstruction. Another related pathology is PA aneurysm associated with end-stage lung disease that doesn't require heart-lung transplantation. Depending on the aneurysm features, donor aorta, autologous recipient tissue, or whole donor PA (if available) can be used for reconstruction (27-29).

\section{Trouble with clamping the hilar vessels}

In addition to the aforementioned issue with clamping a fragile PA, difficulties in applying the clamp around the left atrial cuff can be encountered in a narrow space or with suboptimal exposure. Clamping the left atrial cuff has to be at an optimal angle with enough margins to perform the anastomosis without complications. Slipping of the clamp could occur with inadequate clamp position before finishing the anastomosis and trying to apply another clamp more proximally may not be possible. Dealing with clamping issues is obviously more problematic if the LTx procedure is being performed off pump. In that case, if the clamp can't be applied at an optimal angle, switching to MCS is advised to aid in exposure. Another option is to perform the anastomosis with a no clamp technique using full $\mathrm{CPB}$ with a fibrillating heart induced by rapid ventricular pacing. Mohite $e t a l$. reported favorable outcomes of the no clamp technique in which they use CPB with aortic root vent and maintaining adequate mean arterial pressure to keep the aortic valve closed, without the need for fibrillatory arrest (30). A vent can be placed in the main PA as well to facilitate open anastomosis technique. Another approach to overcome inadequate clamp application on the left atrial cuff is to reunite the two pulmonary veins to create a distal cuff which brings the anastomosis toward the field (31).

\section{Crash on pump}

If the LTx procedure is planned to be conducted off MCS, a backup strategy for cannulation should be in place in case of hemodynamic instability. Adequate preoperative imaging should be obtained to assess calcification and anatomy of the ascending aorta and ilio-femoral vessels. A history of inferior vena cava filter implantation should be noted for its impact on peripheral venous cannulation. Central cannulation is readily accessible though sternotomy and clamshell incisions. The cannulation strategy is more relevant if bilateral thoracotomy incision is planned. In this case, peripheral cannulation is an option as well as centrally through the right thoracotomy. Another alternative for partial pump support is through cannulation of the descending aorta and pulmonary artery on the left side. Decision for cannulation strategy is made based on the urgency of the situation and available access at the time.

\section{Calcified airway}

Although rare, it is important to pay attention for tracheobronchial calcification on preoperative imaging as it may complicate the airway anastomosis during the LTx procedure. Tracheobronchial calcification is an uncommon radiological finding reported to be associated with old age, females, hypercalcemia, and sarcoidosis (32-34). Extensive calcification of the main bronchi may cause fragmentation of the airway cartilage during manipulation, dissection, or performing the anastomosis. Subsequent complication such as air leak or airway dehiscence can be a concern. In such situation, gentle manipulation of the airway is advised. Telescoping technique might be helpful with reinforcement of the anastomosis with overlying soft tissue. Another technical consideration is to run the posterior membranous portion and use interrupted sutures for the anterior cartilaginous wall.

\section{Less common anatomical variants}

\section{Prior pneumonectomy}

History of previous pneumonectomy adds technical 
difficulty to the LTx procedure with altered intrathoracic anatomy due to mediastinal shift. In light of these challenges, LTx procedure after pneumonectomy is relatively uncommon and most of the published reports are of single LTx (35-37). In the Le Pimpec-Barthes et al. series of 14 single LTx, the morbidity and mortality were relatively high due to, in part, post-pneumonectomy syndrome related complications (35). Ganapathi and colleagues reviewed the UNOS database and reported 22 LTx after pneumonectomy (10 bilateral and 12 single) during the period 1999-2011 (36). They observed an increase in the incidence of peri-operative dialysis and 3 -month mortality. Our group approaches these cases on an individual basis with careful preoperative evaluation of their anatomy, surgical history, lung pathology, cardiac function, and cannulation access. When BS-LTx is indicated, our preference for approach is clamshell incision. Special attention is required for cannulation planning for intraoperative MCS when needed, considering the altered mediastinal anatomy (35). To decrease the duration of $\mathrm{CPB}$, one strategy is to implant the post-pneumonectomy side first then perform the contralateral side off CPB (37). Our preferred approach is a single LTx with an over-sized lung, performed via a sternotomy (on MCS). We use controlled reperfusion to minimize injury of the graft and tend to avoid entry into the post-pneumonectomy space to minimize potential contamination of the transplanted side.

\section{Kartagener's syndrome}

Kartagener's syndrome is a rare congenital disease characterized by the triad of chronic sinusitis, bronchiectasis, and situs inversus. Primary ciliary dyskinesia associated with the disease leads to recurrent respiratory infections and end-stage lung disease in some patients for which LTx is indicated $(38,39)$. During the period 1987-2015, only 12 LTx were performed in the United States according to UNOS data reviewed by Hayes and colleagues (38). The rarity of the procedure is attributed to the technical difficulty associated with the situs inversus anatomy. In patients with Kartagener's syndrome, the recipient right main bronchus is long while the left main bronchus is short with early take off of the left upper lobe $(39,40)$. Another technical consideration is the left pulmonary artery anatomy, which is anterior to the bronchus in the recipient with Kartagener's syndrome in contrast to the epi-arterial position in the donor lung $(39,41)$. Certain modifications are required for the LTx procedure to adapt these anatomical alterations. Our approach includes single lumen intubation, toilet bronchoscopy, and then exchange for right-sided double lumen tube. One surgical approach is clamshell incision for optimal exposure with or without intra-operative MCS based on cardiac function, pulmonary artery pressure, hemodynamics and difficulty of the procedure. Alternatively, a sternotomy approach can be utilized with intra-operative MCS (CPB or VA ECMO). We have used this approach with favorable outcomes. When performing the pneumonectomy, we leave the airway stump as long as possible (e.g., transect the left main bronchus just before the upper lobe bronchus). Additionally, we leave the PA stump long and dissected adequately to allow mobility and to facilitate modified anastomosis or reconstruction when needed. On the donor side, the PA is left long as well, while the bronchi are cut short (within one ring of the lobar carina) to minimize airway ischemic complications. The bronchial anastomosis can be performed either as end-to-end or telescoped, based on size matching between the donor and recipient. The left PA anastomosis can be done as end-to-side or end-to-end according to the length and orientation of the vessels $(39,41)$. The atrial cuff anastomosis is done in routine fashion.

\section{Chest wall deformities}

Another technical challenge for LTx is chest wall deformities, such as kyphosis, scoliosis, and pectus excavatum. In addition to concerns for donor-recipient size mismatch and restrictive chest wall compliance which may affect graft function $(42,43)$, chest wall deformities may complicate positioning of the patient during the procedure as well as exposure. Hence, only a few cases of LTx in patients with chest wall deformities are reported (42-45). Our group has reported two cases of bilateral LTx in patients with severe scoliosis, one through clamshell incision and the second through sternotomy (42). Notably, the second case had right-sided airway narrowing secondary to compression by scoliotic spine but did not require intervention. Two groups reported simultaneous bilateral LTx and pectus excavatum repair using the Nuss technique $(43,45)$. The surgical approach was bilateral anterolateral thoracotomies in both cases. These reports suggest that chest wall deformities are not absolute contraindications for LTx; however, such cases need be approached on an individual basis with careful planning for surgical approach 
and cannulation for intra-operative MCS if needed.

\section{Bronchial artery revascularization (BAR)}

Revascularization of the bronchial arteries with en bloc double-lung transplant technique is not part of the standard technique for LTx. This could be due, on one hand, to the complexity of the procedure with concerns for increased ischemic time and BAR thrombosis or stenosis. On the other hand, the current technique of BS-LTx has resulted in a decrease in the incidence of airway complications compared to the initial en bloc technique (without BAR) $(46,47)$. Thus, the experience of the BAR procedure has been limited to few series. The Copenhagen experience $(\mathrm{n}=106$ BAR) showed superior survival rates compared to the ISHLT registry and excellent airway healing (47). These results have encouraged our group to revisit the BAR procedure option through a feasibility study $(46,47)$. The preferred conduit used for revascularization is the internal thoracic artery (less preferably saphenous vein graft for concerns of lower patency) which is harvested before the LTx procedure. On the donor side, the lungs are removed as a block with trachea, esophagus, and descending aorta. During back table preparation of the organ block, the aorta is incised longitudinally along its pleural surface to expose the origin of the intercostal and bronchial arteries. The right intercostobronchial artery and the left bronchial artery (arteries) are identified with the use of a $1 \mathrm{~mm}$ coronary probe. Not all donor blocks are suitable for BAR due to variability in bronchial arterial anatomy or atherosclerosis of the aorta.

En bloc double-LTx with BAR is performed through sternotomy on CPB. The procedure starts with routine preparation of both hila as for BS-LTx. Once the donor lung block is confirmed suitable for BAR, bilateral pneumonectomy is performed and the trachea is divided by removing the carina and main bronchi. The pulmonary arteries are resected to the main pulmonary trunk. The donor lung block is introduced into the chest with each lung placed posterior to the corresponding phrenic nerve (and right atrium on the right) into the pleural spaces. Tracheal anastomosis is performed in the space between the ascending aorta and the superior vena cava. The BAR anastomosis is then performed in end-to-side fashion anastomosing the recipient internal thoracic artery to the orifice of the bronchial artery on the donor descending aorta (sequential anastomosis if needed). The BAR anastomosis is checked by bleeding into the mediastinal tissues of the donor. Main trunk pulmonary anastomosis is done next followed by bilateral left atrial cuff anastomosis $(46,47)$. Arrest of the heart with cardioplegia is not necessary but a short arrest can sometimes facilitate the operation.

Some have advocated for the routine use of en bloc double lung transplantation with BAR as it generally results in excellent airway healing which may improve outcomes. In lieu of routine use, the en bloc double lung transplant technique can prove very useful to address central airway problems or pulmonary artery issues. While we prefer BAR under these conditions, some have advocated for standard en bloc double-lung transplantation without BAR and feel that the tracheal anastomotic issues of earlier years are not as much of an issue due to improved preservation techniques (48).

\section{Concomitant cardiac procedures}

The implementation of the LAS in 2005 has prioritized patients based on the calculated net-transplant benefit, which has resulted in older patients with pulmonary fibrosis and co-morbidities commonly receiving LTx (22). Therefore, a number of patients present for LTx in the current era with associated cardiac pathology that may need to be addressed at time of the transplantation procedure. A few groups have reported their results of concomitant cardiac procedures with LTx; and the outcomes reported are comparable to isolated LTx procedures at experienced centers (49-53). Two aspects need to be planned before the procedure, the surgical approach and the conduct of the operation. The surgical approach varies according to the lung pathology and intended cardiac procedure, as well as the surgeon's preference. Coronary revascularization can be performed through sternotomy, clamshell, or anterolateral thoracotomy $(50,51)$, on CPB or VA ECMO with beating heart, or off pump (54,55). Valve repair or replacement can be performed through sternotomy or clamshell exposure. The conduct of the operation in the majority of reported series is cardiac procedure performed first followed by the LTx procedure. We have generally followed this approach with the exception of CABG where we commonly perform this following lung implantation (to avoid tethering of the internal thoracic artery graft).

With the aim to decrease CPB time, our group has recently used VA ECMO to implant the lung grafts followed by a switch to full CPB to perform the cardiac procedure in selected cases. Our perfusion team developed a protocol that facilitates this approach, which includes using the $\mathrm{CPB}$ 
circuit without the reservoir (closed, VA ECMO circuit) then switching to full $\mathrm{CPB}$ when needed by moving tube clamps and using the reservoir. This approach is particularly useful when addressing functional tricuspid regurgitation in the setting of pulmonary hypertension without significant annular dilation or leaflet tethering, whereas the tricuspid valve can be reassessed after finishing LTx procedure with improvement of pulmonary artery pressure and the degree of tricuspid regurgitation. Similarly, if arrhythmia ablation surgery is planned, we perform this after the LTx as the procedure per se includes surgical pulmonary vein isolation, then we either excise or clip the left atrial appendage and complete the right atrial lesions.

\section{Delayed chest closure}

The strategy of delayed chest closure has been adopted by several LTx groups to address intra-operative complications such as coagulopathy, significant pulmonary edema, or hemodynamic instability when chest closure is attempted (56-59). Our technique for delayed chest closure includes negative pressure wound therapy using a foam sponge then sealed by adhesive dressing or occasionally sponge packing and covering of the incision with a latex membrane that is stapled to the skin (59). The sternal edges or ribs are retracted with a plastic or metal bridge. Standard chest tubes are inserted for drainage. The patient is kept on broad spectrum antimicrobial coverage while the chest is opened. Timing for formal chest closure relies on improvement of pulmonary edema, hemodynamics, or bleeding. Other modalities are temporary skin closure with running suture and using sternal or rib retractor $(57,58)$. In a matched cohort of primary closure, Shigemura et al. (57) have shown the delayed chest closure resulted in decrease incidence of severe primary graft dysfunction. There was no increase incidence of wound infection in our group's recent report (59) and the Force et al. series (56); however, there was an increased hospital stay and morbidity. In contrast, Aguilar $e t$ al. have reported increased incidence of wound infection (58).

\section{Comment}

The LTx field has witnessed major progress in the surgical techniques over the last three decades with marked improvement in the outcomes. Currently, BS-LTx is the most common approach performed by most surgical groups for the superior long-term outcomes compared to single LTx. Additionally, the en bloc double-lung transplant technique can be used effectively to treat central airway and pulmonary artery issues. Our group is investigating the BAR en bloc double-lung technique, which is a technically more demanding operation, but outcomes could be rewarding. The surgical approach for BS-LTx varies based on the surgeon preference and recipient-related factors. In the absence of routine VA ECMO use intra-operatively, as some have advocated, it is important to have a plan for intra-operative MCS in case it is needed. Our preference is to perform the LTx procedure off pump whenever feasible and, if intra-operative MCS is required, we prefer to use VA ECMO. Lastly, another important aspect of any surgical technique is the ability to teach trainees and reproducibility by junior surgeons. In our experience, using a standard approach for dissection and mobilizing the hilar structures as well as the anastomosis technique facilitates the learning process. Adequate exposure and optimal field set-up are key factors for a smooth conduct of the operation, and in that sense, the clamshell approach (with or without VA ECMO support) might be an advised approach for junior surgeons starting their LTx practice.

\section{Acknowledgments}

Funding: None.

\section{Footnote}

Provenance and Peer Review: This article was commissioned by the Guest Editor (Jonathan D'Cunha) for the series "Lung Transplantation: Past, Present, and Future" published in Fournal of Thoracic Disease. The article has undergone external peer review.

Conflicts of Interest: All authors have completed the ICMJE uniform disclosure form (available at: http:// dx.doi.org/10.21037/jtd-2021-22). The series "Lung Transplantation: Past, Present, and Future" was commissioned by the editorial office without any funding or sponsorship. KRM reports other from Lung Bioengineering, outside the submitted work. The other authors have no conflicts of interest to declare.

Ethical Statement: The authors are accountable for all aspects of the work in ensuring that questions related to the accuracy or integrity of any part of the work are appropriately investigated and resolved. 
Open Access Statement: This is an Open Access article distributed in accordance with the Creative Commons Attribution-NonCommercial-NoDerivs 4.0 International License (CC BY-NC-ND 4.0), which permits the noncommercial replication and distribution of the article with the strict proviso that no changes or edits are made and the original work is properly cited (including links to both the formal publication through the relevant DOI and the license). See: https://creativecommons.org/licenses/by-nc-nd/4.0/.

\section{References}

1. Patterson GA, Cooper JD, Goldman B, et al. Technique of successful clinical double-lung transplantation. Ann Thorac Surg 1988;45:626-33.

2. Toronto Lung Transplant Group. Unilateral lung transplantation for pulmonary fibrosis. N Engl J Med 1986;314:1140-5.

3. Pasque MK, Cooper JD, Kaiser LR, et al. Improved technique for bilateral lung transplantation: rationale and initial clinical experience. Ann Thorac Surg 1990;49:785-91.

4. Hayes D, Cherikh WS, Chambers DC, et al. The International Thoracic Organ Transplant Registry of the International Society for Heart and Lung Transplantation: Twenty-second pediatric lung and heart-lung transplantation report-2019; Focus theme: Donor and recipient size match. J Heart Lung Transplant 2019;38:1015-27.

5. Yusen RD, Shearon TH, Qian Y, et al. Lung transplantation in the United States, 1999-2008. Am J Transplant 2010;10:1047-68.

6. Meyers BF, Sundaresan RS, Guthrie T, et al. Bilateral sequential lung transplantation without sternal division eliminates posttransplantation sternal complications. J Thorac Cardiovasc Surg 1999;117:358-64.

7. Boasquevisque CHR, Yildirim E, Waddel TK, et al. Surgical techniques: lung transplant and lung volume reduction. Proc Am Thorac Soc 2009;6:66-78.

8. Venuta F, Rendina EA, De Giacomo T, et al. Bilateral sequential lung transplantation without sternal division. Eur J Cardiothorac Surg 2003;23:894-7.

9. Aigner C, Klepetko W. Bilateral Lung Transplantation. Oper Tech Thorac Cardiovasc Surg 2012;17:181-93.

10. Elde S, Huddleston S, Jackson S, et al. Tailored Approach to Surgical Exposure Reduces Surgical Site Complications after Bilateral Lung Transplantation. Surg Infect (Larchmt) 2017;18:929-35
11. Mody GN, Coppolino A, Singh SK, et al. Sternotomy versus thoracotomy lung transplantation: key tips and contemporary results. Ann Cardiothorac Surg 2020;9:60-4.

12. Gust L, D'Journo X-B, Brioude G, et al. Single-lung and double-lung transplantation: technique and tips. J Thorac Dis 2018;10:2508-18.

13. Davis RD. Bilateral Sequential Lung Transplantation. Oper Tech Thorac Cardiovasc Surg 2007;12:57-72.

14. Balsara KR, Krupnick AS, Bell JM, et al. A single-center experience of 1500 lung transplant patients. J Thorac Cardiovasc Surg 2018;156:894-905.e3.

15. Laporta Hernandez R, Aguilar Perez M, Lázaro Carrasco MT, et al. Lung Transplantation in Idiopathic Pulmonary Fibrosis. Med Sci (Basel) 2018;6:68.

16. George PM, Patterson CM, Reed AK, et al. Lung transplantation for idiopathic pulmonary fibrosis. Lancet Respir Med 2019;7:271-82.

17. Siddiqui FM, Diamond JM. Lung transplantation for chronic obstructive pulmonary disease: past, present, and future directions. Curr Opin Pulm Med 2018;24:199-204.

18. Mitchell AB, Glanville AR. Lung transplantation: a review of the optimal strategies for referral and patient selection. Ther Adv Respir Dis 2019;13:1753466619880078.

19. Hayanga JWA, D'Cunha J. The surgical technique of bilateral sequential lung transplantation. J Thorac Dis 2014;6:1063-9.

20. Conte JV, Borja MJ, Patel CB, et al. Lung transplantation for primary and secondary pulmonary hypertension. Ann Thorac Surg 2001;72:1673-9; discussion 1679-80.

21. George MP, Champion HC, Pilewski JM. Lung transplantation for pulmonary hypertension. Pulm Circ 2011;1:182-91.

22. McCurry KR. Brief Overview of Lung, Heart, and HeartLung Transplantation. Crit Care Clin 2019;35:1-9.

23. Shigemura N, Bhama J, Gries CJ, et al. Lung transplantation in patients with prior cardiothoracic surgical procedures. Am J Transplant 2012;12:1249-55.

24. McKellar SH, Bowen ME, Baird BC, et al. Lung transplantation following coronary artery bypass surgeryimproved outcomes following single-lung transplant. J Heart Lung Transplant 2016;35:1289-94.

25. Omara M, Okamoto T, Arafat A, et al. Lung transplantation in patients who have undergone prior cardiothoracic procedures. J Heart Lung Transplant 2016;35:1462-70.

26. Sakamaki $Y$, Minami M, Ohta $M$, et al. Pulmonary artery dissection complicating lung transplantation for 
primary pulmonary hypertension. Ann Thorac Surg 2006;81:360-2.

27. Force SD, Lau CL, Moazami N, et al. Bilateral lung transplantation and pulmonary artery reconstruction in a patient with chronic obstructive pulmonary disease and a giant pulmonary artery aneurysm. J Thorac Cardiovasc Surg 2003;126:864-6.

28. Zanotti G, Hartwig MG, Davis RD. A simplified technique for pulmonary artery aneurysm repair in a lung transplant recipient with right ventricular outflow tract obstruction. J Thorac Cardiovasc Surg 2013;145:295-6.

29. Noda M, Okada Y, Saiki Y, et al. Reconstruction of pulmonary artery with donor aorta and autopericardium in lung transplantation. Ann Thorac Surg 2013;96:e17-9.

30. Mohite PN, Garcia-Saez D, Sabashnikov A, et al. Noclamp technique for pulmonary artery and venous anastomoses in lung transplantation. J Heart Lung Transplant 2014;33:1133-8.

31. Robert JH, Murith N, de Perrot M, et al. Lung transplantation: how to perform the venous anastomosis when clamping is too distal. Ann Thorac Surg 2000;70:2164-5.

32. Agrawal A, Kumar SS, Raghu K, et al. Incidental tracheobronchial calcification. Medical Journal of Dr DY Patil University 2012;5:166.

33. Wright J, Jones E. Diffuse Calcification of the Airways. Mod Pathol 2001;14:717-9.

34. Bansal S, Utpat K, Desai U, et al. Sarcoidosis Presenting with Tracheobronchial Calcification and Nodularity: An Unusual Case Presentation with Treatment Response Assessment by 18F-FDG-PET/CT. Indian J Nucl Med 2017;32:217-20.

35. Le Pimpec-Barthes F, Thomas P-A, Bonnette P, et al. Single-lung transplantation in patients with previous contralateral pneumonectomy: technical aspects and results. Eur J Cardiothorac Surg 2009;36:927-32.

36. Ganapathi AM, Speicher PJ, Castleberry AW, et al. The effect of prior pneumonectomy or lobectomy on subsequent lung transplantation. Ann Thorac Surg 2014;98:1922-8; discussion 1928-9.

37. Ris HB, Krueger T, Gonzalez M, et al. Successful bilateral lung transplantation after previous pneumonectomy. Ann Thorac Surg 2011;91:1302-4.

38. Hayes D, Reynolds SD, Tumin D. Outcomes of lung transplantation for primary ciliary dyskinesia and Kartagener syndrome. J Heart Lung Transplant 2016;35:1377-8

39. Gauthier JM, Takahashi T, Bierhals AJ, et al. Technical
Considerations for Lung Transplantation in Kartagener's Syndrome. Ann Thorac Surg 2019;107:e337-9.

40. Macchiarini P, Chapelier A, Vouhé P, et al. Double lung transplantation in situs inversus with Kartagener's syndrome. Paris-Sud University Lung Transplant Group. J Thorac Cardiovasc Surg 1994;108:86-91.

41. Mentzer SJ, Aranki SF, Reilly JJ, et al. Single-lung transplantation in situs inversus. Ann Thorac Surg 1994;58:1176-8.

42. Su JW, Mason DP, Murthy SC, et al. Successful double lung transplantation in 2 patients with severe scoliosis. J Heart Lung Transplant 2008;27:1262-4.

43. Valdivia D, Kamler M, Aigner C. Bilateral Lung Transplantation and Simultaneous Pectus Excavatum Correction Using the Nuss Technique. Ann Thorac Surg 2019;107:e275-7.

44. Fukahara K, Minami K, Hansky B, et al. Successful heartlung transplantation in a patient with kyphoscoliosis. J Heart Lung Transplant 2003;22:468-73.

45. Zhang Z, Ding H, Liu F, et al. Successful bilateral lung transplantation and simultaneous Nuss technique correction of pectus excavatum post-allogeneic haematopoietic stem cell transplantation. Interact Cardiovasc Thorac Surg 2020;30:319-20.

46. Tong MZ, Johnston DR, Pettersson GB. The role of bronchial artery revascularization in lung transplantation. Thorac Surg Clin 2015;25:77-85.

47. Yun JJ, Unai S, Pettersson G. Lung transplant with bronchial arterial revascularization: review of surgical technique and clinical outcomes. J Thorac Dis 2019;11:S1821-8.

48. Anraku M, Volling C, Pierre A. Remembering en bloc double-lung transplantation. J Heart Lung Transplant 2011;30:485-6.

49. Johnson SB, Allred AM, Cline AM, et al. Cardiac procedures in lung transplant recipients do not increase mortality in selected patients. Ann Thorac Surg 2006;82:460-3; discussion 463-64.

50. Parekh K, Meyers BF, Patterson GA, et al. Outcome of lung transplantation for patients requiring concomitant cardiac surgery. J Thorac Cardiovasc Surg 2005;130:859-63.

51. Castleberry AW, Martin JT, Osho AA, et al. Coronary revascularization in lung transplant recipients with concomitant coronary artery disease. Am J Transplant 2013;13:2978-88.

52. Biniwale R, Ross D, Iyengar A, et al. Lung transplantation and concomitant cardiac surgery: Is it justified? J Thorac 
Cardiovasc Surg 2016;151:560-6.

53. Niikawa H, Okamoto T, Thuita L, et al. Outcomes of Lung Transplantation for Patients Undergoing Previous or Simultaneous Cardiac Surgery. J Heart Lung Transplant 2018;37:S164-5.

54. Ueyama K, Miyahara S, Ide Y, et al. On-pump beating CABG concomitant with bilateral living-donor lobar lung transplantation. Heart Lung 2019;48:166-8.

55. Do YW, Jung HS, Lee CY, et al. Combined Bilateral Lung Transplantation and Off-Pump Coronary Artery Bypass. Korean J Thorac Cardiovasc Surg 2016;49:461-4.

56. Force SD, Miller DL, Pelaez A, et al. Outcomes of delayed

Cite this article as: Elgharably H, Javorski MJ, McCurry KR. Bilateral sequential lung transplantation: technical aspects. J Thorac Dis 2021;13(11):6564-6575. doi: 10.21037/jtd-2021-22 chest closure after bilateral lung transplantation. Ann Thorac Surg 2006;81:2020-4; discussion 2024-5.

57. Shigemura N, Orhan Y, Bhama JK, et al. Delayed chest closure after lung transplantation: techniques, outcomes, and strategies. J Heart Lung Transplant 2014;33:741-8.

58. Aguilar PR, Bemiss BC, Witt C, et al. Impact of Delayed Chest Closure on Surgical Site Infection After Lung Transplantation. Ann Thorac Surg 2017;104:1208-14.

59. Rafiroiu S, Hassouna H, Ahmad U, et al. Consequences of Delayed Chest Closure During Lung Transplantation. Ann Thorac Surg 2020;109:277-84. 